\title{
THERMOPOWER OF THE KONDO LATTICE
}

\author{
M. LAVAGNA, C. LACROIX and M. CYROT \\ Laboratoire Louis Néel ${ }^{1}$, CNRS, 166 X, 38042 Grenoble-Cedex, France
}

Received 10 February 1982

\begin{abstract}
The thermopower of a Kondo lattice has been calculated within the same model as used earlier for the resistivity: the Kondo interaction is treated as a fictitious $s-f$ hybridization for which both the thermal and spatial fluctuations are considered. The thermopower has been found to take giant values at low temperatures with a peak roughly at the same temperature $T_{1}$ as that of the resistivity. Its sign can be either positive or negative depending on the number of conduction electrons
\end{abstract}

1. Introduction. Among the various Kondo anomalies observed in concentrated alloys of anomalous rare earths, the giant thermopower $S(T)$ is perhaps the most spectacular one. A broad peak can be observed in all these compounds, of which the maximum may be as large as $40 \mu \mathrm{V} \mathrm{K}^{-1}$. It occurs roughly at the same temperature as the maximum of the resistivity. The sign of the thermopower at low temperatures can be either positive in systems like $\mathrm{CeCu}_{2} \mathrm{Si}_{2}$ [1] and $\mathrm{CeAl}_{2}$ [2], or negative in $\mathrm{CeAl}_{3}$ [3]. Two main theories exist to explain these results. Peschel and Fulde [4] considered a system of two nonmagnetic singlets separated by an energy $\delta$. They got a contribution to $S$ by inelastic exchange scattering which has an extremum at $T \approx 0.3 \delta$. Depending on the sign of the scattering potential $V$, the thermopower may show a maximum or a minimum. Bhattacharjee and Coqblin [5] calculated the thermo. power within the model introduced by CornutCoqblin [6] for cerium in order to take into account the orbital degeneracy and the influence of the crystalline field on the Kondo effect. They obtained giant values of $S$ even if the scattering potential $V$ is equal to zero, contrary to the results of Peschel and Fulde. The thermopower has a peak at a temperature between $\delta / 6$ and $\delta / 3$. The nature of the peak (maximum or minimum) depends not only on the ratio $V / J$ as

\footnotetext{
1 Laboratoire propre du CNRS associé à l'Université Scientifique et Médicale de Grenoble.
}

was the case in ref. [4] ( $J$ is the usual $\mathrm{s}-\mathrm{f}$ exchange scattering). Both studies show the effect of the crystal field on the thermopower in a one-impurity Kondo model and do not take into account the effect of the lattice. The purpose of this paper is to study the thermopower in a Kondo lattice. The starting point is the effective hamiltonian of Lacroix and Cyrot [7] which is obtained by the transformation of the Kondo exchange interaction into a fictitious $s-f$ hybridization within a functional integration method:

$$
\begin{aligned}
H & =\sum_{k \sigma}\left(\epsilon_{k}+J / 4\right) C_{k \sigma}^{+} C_{k \sigma}+\sum_{i \sigma}\left(E_{0}+J n / 4\right) d_{i \sigma}^{+} d_{i \sigma} \\
& +\frac{1}{2} J \sum_{i} x_{i}\left(d_{i \uparrow}^{+} C_{i \uparrow}+C_{i \downarrow}^{+} d_{i \downarrow}\right) \\
& +\frac{1}{2} J \sum_{i} y_{i}\left(d_{i \downarrow}^{+} C_{i \downarrow}+C_{i \uparrow}^{+} d_{i \uparrow}\right)
\end{aligned}
$$

where $C_{k}^{+}, d_{i}^{+}$and $\epsilon_{k}, E_{0}$ are the creation operators and the energies of an s electron of wave vector $k$ and an f electron on site $i$, respectively, $J$ is the usual Kondo $\mathrm{s}-\mathrm{f}$ interaction, $n$ is the number of conduction electrons per atom, $x_{i}$ and $y_{i}$ represent the fictitious $\mathrm{s}-\mathrm{f}$ hybridization.

In this model, a gap of width $T_{\mathrm{K}}$ occurs in the density of states. This gap is located around the impurity level $E_{0}$.

We review in section 2 the different stages of the electrical conductivity calculation in this model, de- 
tailed in a previous paper [8] and show the importance of the introduction of thermal and spatial fluctuations in the lattice. Once the expression of the conductivity $\sigma(\omega)$ has been established, the thermopower can be produced easily, see section 3 .

2. Electrical conductivity of the Kondo lattice, a brief review. In order to account for the large decrease in the resistivity well above $T_{\mathrm{K}}$, it is necessary to go beyond a mean field theory and to introduce thermal fluctuations of the parameter $x$. At high temperatures, these are evaluated as

$\left\langle x^{2}\right\rangle=k_{\mathrm{B}} T /\left[\left(J^{2} / 2 D\right) \log \left(T / T_{\mathrm{K}}\right)\right]$.

At high temperatures, the system can be considered as a collection of incoherent impurities. The electrical resistivity can be evaluated by means of the phase shift $\phi(\omega)$ :

$\rho(\omega) \sim \sin ^{2} \phi(\omega), \phi(\omega)=\operatorname{arctg}\left[\pi\left(J x^{2} / 2\right) / 2 D \omega\right]$,

and, finally, taking into account the thermal fluctuations:

$\rho \sim(J / 2 D)^{2}\left[1+(J / D) \log \left(k_{\mathrm{B}} T / D\right)\right]$.

We recognize here the usual Kondo behaviour at high temperatures with a logarithmic decrease in $J$ $\log \left(k_{\mathrm{B}} T / D\right)$.

At low temperatures, spatial fluctuations from site to site are taken to be responsible for the electron scattering:

$V=\frac{1}{2} J \delta_{i} \sum_{i}\left(d_{i \uparrow}^{+} C_{i \uparrow}+C_{i \downarrow}^{+} d_{i \downarrow}+C_{i \uparrow}^{+} d_{i \uparrow}+d_{i \downarrow}^{+} C_{i \downarrow}\right)$,

$\delta_{i}=x_{i}-\left\langle x_{i}\right\rangle$.

The average value $\left\langle\delta^{2}\right\rangle$ is evaluated to be $k T / 2|J|$ and the electrical conductivity is calculated in a Boltzmann theory for $n<1$ :

$$
\begin{aligned}
& \sigma(\omega)=\left(4 e^{2} / 3 m^{*}\right)(J \delta / 2)^{-2}(J x / 2)^{-2}\left(\omega_{2}-\omega\right) \\
& \quad \times\left(\omega-E_{0}-J n / 4\right)^{2},
\end{aligned}
$$

$\omega_{2}$ being the lower limit of the gap in the density of states. For $n \neq 1$, the resistivity is of the metallic type, increasing with temperature and exhibits a maximum at $T_{1} \approx(1-n)^{1 / 3} T_{\mathrm{K}}$. For $n=1$, the resistivity is typically that of a semi-conductor with a tempera. ture behaviour of $\exp \left(T_{\mathrm{K}} / T\right)$.
3. Thermopower of the Kondo lattice. The thermopower can be deduced from the expression of the conductivity by the usual formula:

$$
\begin{gathered}
S(T)=\frac{1}{e T} \int\left(-\frac{\mathrm{d} f}{\mathrm{~d} \omega}\right)\left(\omega-E_{\mathrm{F}}\right) \sigma(\omega) \mathrm{d} \omega \\
\times\left[\int\left(-\frac{\mathrm{d} f}{\mathrm{~d} \omega}\right) \sigma(\omega) \mathrm{d} \omega\right]^{-1},
\end{gathered}
$$

where $e$ is the negative electron charge.

At low temperature and for $n<1$, we can write ${ }^{\circ}$ using eq. (5):

$S(T)=S(0)\left[1+\left(T / T_{2}\right)^{2}\right] /\left[1+\left(T / T_{1}\right)^{2}\right]$,

where

$$
\begin{aligned}
& S(0)=-\left(2 \pi^{2} k_{\mathrm{B}} / 3 e\right)\left[\frac{3}{4} \pi(1-n)\right]^{-2 / 3}\left(T / T_{\mathrm{K}}\right), \\
& T_{1}=0.367 T_{\mathrm{K}}(1-n)^{1 / 3}, \quad T_{2}=0.269 T_{\mathrm{K}} .
\end{aligned}
$$

As $n$ is very close to 1 in almost all these compounds, the thermopower takes giant positive values at low temperatures as a result of the factor $(1-n)^{-2 / 3}$. On the other hand, $T_{1} \ll T_{2}$ generally and the thermo-

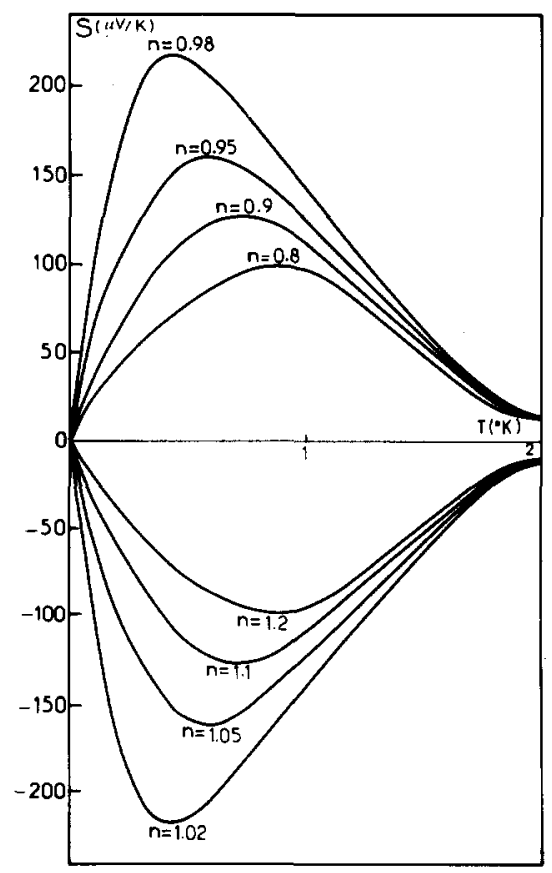

Fig. 1. 
power exhibits a maximum at a temperature slightly higher than $T_{1}$, the temperature of the maximum of the resistivity.

It is easy to transpose this to the case $n>1$ :

$S(0)=\left(2 \pi^{2} k_{\mathrm{B}} / 3 e\right)\left[\frac{3}{4} \pi(n-1)\right]^{-2 / 3}\left(T / T_{\mathrm{K}}\right)$.

The thermopower takes again giant values but of negative sign with a peak slightly above $T_{1}^{\prime}=0.367 T_{\mathrm{K}}(n$ $-1)^{1 / 3}$. So, the sign of $(n-1)$ determines the nature (maximum or minimum) of the peak of the thermopower. The experimental cases $\mathrm{CeCu}_{2} \mathrm{Si}_{2}, \mathrm{CeAl}_{2}$ and $\mathrm{CeAl}_{3}$ probably correspond to each of these two possibilities. The experimental results of Jaccard [9] about the variation of $S(300 \mathrm{~K})$ with $x$ in $\mathrm{Tm}_{x}$ Se confirm that a change in the sign of the thermopower occurs near the stoichiometry (that is $n=1$ ). At high temperatures, the use of eq. (2) yields a thermopower equal to zero. This result is obvious since we are drawn to the original Kondo theory without scattering potential $V$. In order to find a result different from zero we might introduce this potential $V$ in the calculation. In fact, this does not seem to be very interesting because there is no general behaviour of the thermopower at high temperatures in these systems. Fig. 1 shows the schematic thermal variation of the thermopower $S(T)$ for various $n$. This describes qualitatively the experimental behaviour of $S(T)$ with giant values at low temperatures of positive or negative sign depend ing on the compound studied. The high temperature peaks which are often observed in the compounds can be explained by crystal field effects as was shown by Bhattacharjee and Coqblin [5]. Our model gives a qualitative description for the low temperature peak.

\section{References}

[1] W. Franz, F. Steglich and D. Wohlleben, J. de Phys. Suppl. no. 5, 40 (1979) C5-342.

[2] J.H. Moeser, F. Steglich and G.V. Minnigerode, J. Low Temp. Phys. 15 (1974) 91.

[3] P.B. van Aken, H.J. van Daal and K.H.J. Buschow, Phys. Lett. 49A (1974) 201.

[4] I. Peschel and P. Fulde, Z. Phys. 238 (1970) 99.

[5] A.K. Bhattacharjee and B. Coqblin, Phys. Rev. B13 (1976) 3441.

[6] B. Cornut and B. Coqblin, Phys. Rev. B5 (1972) 4541.

[7] C. Lacroix and M. Cyrot, Phys. Rev. B20 (1979) 1969.

[8] M. Lavagna, C. Lacroix and M. Cyrot, J. Phys. F12 (1982), to be published.

[9] D. Jaccard and J. Sierro, Helv. Phys. Acta (1982), to be published. 